\title{
Scale-Free Property for Degrees and Weights in a Preferential Attachment Random Graph Model
}

\author{
István Fazekas and Bettina Porvázsnyik \\ Faculty of Informatics, University of Debrecen, P.O. Box 12, Debrecen 4010, Hungary \\ Correspondence should be addressed to István Fazekas; fazekasi@inf.unideb.hu
}

Received 8 April 2013; Accepted 1 August 2013

Academic Editor: Shein-chung Chow

Copyright (C) 2013 I. Fazekas and B. Porvázsnyik. This is an open access article distributed under the Creative Commons Attribution License, which permits unrestricted use, distribution, and reproduction in any medium, provided the original work is properly cited.

\begin{abstract}
A random graph evolution mechanism is defined. The evolution studied is a combination of the preferential attachment model and the interaction of four vertices. The asymptotic behaviour of the graph is described. It is proved that the graph exhibits a power law degree distribution; in other words, it is scale-free. It turns out that any exponent in $(2, \infty)$ can be achieved. The proofs are based on martingale methods.
\end{abstract}

\section{Introduction}

During the last 15 years the behaviour of many types of real-world networks was investigated. Such networks are the WWW, the Internet, and social and biological networks (see [1] for an overview). The main common characteristic of such networks is their scale-free nature, in other words the power law degree distribution, that is, $p_{k} \sim C k^{-\gamma}$, as $k \rightarrow$ $\infty$. Using real-life data, the exponents were determined for several cases. For the WWW the in-degree and the out-degree of web pages follow power law with $\gamma_{\text {in }}=2.1$ and $\gamma_{\text {out }}=2.7$, for the Internet $\gamma=2.3$, for the movie actor network $\gamma=2.3$, and for the collaboration graph of mathematicians $\gamma=2.4$ (see [1] for details). To describe the phenomenon, in [2] the preferential attachment model was introduced. In that model the growing procedure of the random graph is the following. At every time $t=2,3, \ldots$ a new vertex with $m$ edges is added so that the edges link the new vertex to $m$ existing vertices. The probability $\pi_{i}$ that the new vertex will be connected to the old vertex $i$ depends on the degree $k_{i}$ of vertex $i$, so that $\pi_{i}=k_{i} / \sum_{j} k_{j}$. Then several papers were devoted to the proof of the power law in the preferential attachment model (see, e.g., [3]).

There are versions of the preferential attachment model $($ see $[4,5])$. It turned out that besides the degrees of the vertices other characteristics of the graph can be important (see [5]). In [6] a model based on the interaction of three vertices was introduced. Then the power law degree distribution in that model was obtained in [7].

In this paper, we extend the model and the results of $[6,7]$ to interactions of four vertices. Our model is the following. For short, a complete graph with four vertices we will call tetragon. The starting point at time $n=0$ is one tetragon. The initial weight of this graph is one. This graph contains 4 vertices, 6 edges, and 4 triangles. Each of these objects has initial weight 1 . After the initial step we start to increase the size of the graph. The main feature of the procedure is that at each step $n=1,2, \ldots$ we consider four vertices and draw all nonexisting edges between these vertices. So we obtain a tetragon. The weight of this tetragon and the weights of all the objects in the tetragon are increased by 1 . (That is, we increase the weights of 4 vertices, 6 edges, 4 triangles, and 1 tetragon.) The choice of the four vertices is the following.

There are two possibilities at each step. With probability $p$ we add a new vertex that interacts with three old vertices, on the other hand, with probability $(1-p)$ four old vertices interact. Here $0<p \leq 1$ is fixed.

When we add a new vertex, then we choose 3 old vertices and they together will form a tetragon. However, to choose the three old vertices we have two possibilities. With probability $r$ we choose a triangle from the existing triangles according to the weights of the triangles. It means that a triangle of weight $w_{t}$ is chosen with probability $w_{t} / \sum_{h} w_{h}$ (preferential attachment rule). On the other hand, with 
probability $1-r$, we choose among the existing vertices uniformly; that is, all three vertices have the same chance.

When four old vertices interact, we have again two possibilities. With probability $q$, we choose among the existing tetragons according to their weights. It means that a tetragon of weight $\widetilde{w}_{t}$ is chosen with probability $\widetilde{w}_{t} / \sum_{h} \widetilde{w}_{h}$ (preferential attachment rule). On the other hand, with probability $1-q$, we choose among the existing vertices uniformly; that is, all four vertices have the same chance.

Our aim is to prove that the above mechanism produces a scale-free graph. We follow the lines of $[6,7]$. Let $X(n, d, w)$ denote the number of vertices of weight $w$ and degree $d$ after the $n$th step. Let $V_{n}$ denote the number of vertices after the $n$th step. Let $\mathscr{F}_{n-1}$ denote the $\sigma$-algebra of observable events after the $(n-1)$ th step. First we calculate the conditional expectation $\mathbb{E}\left\{X(n, d, w) \mid \mathscr{F}_{n-1}\right\}$, see Lemma 2. Then we prove (Theorem 3 ) that $X(n, d, w) / V_{n} \rightarrow x_{d, w}$ almost surely (a.s.) as $n \rightarrow \infty$, where $x_{d, w}$ are fixed nonnegative numbers. The main tool of the proof is the Doob-Meyer decomposition of submartingales. We remark that in the 3-interaction model of [6] the limit $x_{d, w}$ is always positive (see [7]). As in our case the limit can be zero, we should modify the proof presented in [7].

We show that $x_{d, w}, d=3,4, \ldots, 3 w, w=1,2, \ldots$, is a proper two-dimensional discrete probability distribution (Lemma 4). Then we turn to the scale-free property for the weights. Let $X(n, w)$ denote the number of vertices of weight $w$ after the $n$th step. Then for all $w=1,2, \ldots$ we have

$$
\frac{X(n, w)}{V_{n}} \longrightarrow x_{w}=x_{3, w}+x_{4, w}+\cdots+x_{3 w, w}
$$

almost surely and $x_{w} \sim C w^{-(1+1 / \alpha)}$, as $w \rightarrow \infty$ (Theorem 5). To derive the above results from Theorem 3, we need only some known facts about the $\Gamma$-function, see Lemma 4 and Theorem 5. Finally, we obtain the scale-free property for the degrees. Let us denote by $U(n, d)$ the number of vertices of degree $d$ after the $n$th step. For any $d \geq 3$ we have

$$
\frac{U(n, d)}{V_{n}} \longrightarrow u_{d}
$$

almost surely as $n \rightarrow \infty$, where $u_{d}$ are positive numbers. Furthermore,

$$
u_{d} \sim \frac{\Gamma(1+(\beta+1) / \alpha)}{\alpha_{2} \Gamma(1+\beta / \alpha)}\left(\frac{\alpha d}{\alpha_{2}}\right)^{-(1+1 / \alpha)},
$$

as $d \rightarrow \infty$, where $\alpha, \beta$, and $\alpha_{2}$ are appropriate constants (see Theorem 8). In both cases the exponent is $1+1 / \alpha$. We can see that its value can be any number in $(2, \infty)$.

If we compare the results and methods of the present paper with the ones in $[6,7]$, we can see that the calculations for the 4-vertice model are much longer than those of the 3vertice model (see the proofs of Lemma 2 and Theorem 3 ). One can think that when we want to extend our model to the interaction of $N$ vertices, then formulae will be burdening. But it is not the case. Analysing the proof of Theorem 3, it turns out that several terms are asymptotically negligible and this phenomenon remains true for the $N$-vertices model, too. Therefore the asymptotic results of the 3 - and 4 -vertice models can be extended to the $N$-vertices model. Moreover, several versions of the $N$-vertices model can be constructed and their fit to real-life data can be studied.

\section{The Evolution of the Graph}

Throughout the paper $0<p \leq 1,0 \leq r \leq 1,0 \leq q \leq 1$ are fixed numbers. Let $X(n, d, w)$ denote the number of vertices of weight $w$ and degree $d$ after the $n$th step. Let $V_{n}$ denote the number of vertices after the $n$th step.

Remark 1. Each vertex has initial weight 1 and initial degree 3. When a vertex takes part in an interaction, then its weight is increased by 1 and its degree may be increased by $0,1,2$, or 3 . Therefore $X(n, d, w)$ can be positive only for $1 \leq w$ and $3 \leq d \leq 3 w$.

Let $\mathscr{F}_{n-1}$ denote the $\sigma$-algebra of observable events after the $(n-1)$ th step. We compute the conditional expectation of $X(n, d, w)$ with respect to $\mathscr{F}_{n-1}$ for $w \geq 1$.

Let

$$
\begin{gathered}
\alpha_{1}=(1-p) q, \quad \alpha_{2}=\frac{3}{4} p r \\
\alpha=\alpha_{1}+\alpha_{2}, \\
\beta=3(1-r)+\frac{4(1-p)(1-q)}{p} .
\end{gathered}
$$

The following lemma contains the basic equation of the paper.

Lemma 2. We have

$$
\begin{aligned}
\mathbb{E}\{X & \left.(n, d, w) \mid \mathscr{F}_{n-1}\right\} \\
= & X(n-1, d, w)\left[1-\left(\frac{w}{n} \alpha+\frac{p}{V_{n-1}} \beta\right)\right] \\
+ & X(n-1, d, w-1) \\
\times & {\left[(1-p)\left(q \frac{w-1}{n}+(1-q) \frac{\left(\begin{array}{c}
d \\
3
\end{array}\right)}{\left(\begin{array}{c}
V_{n-1} \\
4
\end{array}\right)}\right)\right] } \\
+ & X(n-1, d-1, w-1) \\
\times & {\left[p\left(r \frac{3(w-1)}{4 n}+(1-r) \frac{\left(\begin{array}{c}
d-1 \\
2
\end{array}\right)}{\left(\begin{array}{c}
V_{n-1} \\
3
\end{array}\right)}\right)\right.} \\
& +(1-p)(1-q) \frac{\left(\begin{array}{c}
d-1 \\
2
\end{array}\right)\left(\begin{array}{c}
\left.V_{n-1}-d\right) \\
\left(\begin{array}{c}
V_{n-1} \\
4
\end{array}\right)
\end{array}\right]}{+} X(n-1, d-2, w-1)
\end{aligned}
$$




$$
\begin{aligned}
& \times\left[p(1-r) \frac{(d-2)\left(V_{n-1}-d+1\right)}{\left(\begin{array}{c}
V_{n-1} \\
3
\end{array}\right)}\right. \\
& \left.+(1-p)(1-q) \frac{(d-2)\left(\begin{array}{c}
V_{n-1}-d+1 \\
2
\end{array}\right)}{\left(\begin{array}{c}
V_{n-1} \\
4
\end{array}\right)}\right] \\
& +X(n-1, d-3, w-1) \\
& \times\left[p(1-r) \frac{\left(\begin{array}{c}
V_{n-1}-d+2 \\
2
\end{array}\right)}{\left(\begin{array}{c}
V_{n-1} \\
3
\end{array}\right)}\right. \\
& \left.+(1-p)(1-q) \frac{\left(\begin{array}{c}
V_{n-1}-d+2 \\
3
\end{array}\right)}{\left(\begin{array}{c}
V_{n-1} \\
4
\end{array}\right)}\right]+p \delta_{d, 3} \delta_{w, 1}
\end{aligned}
$$

for $w \geq 1$ and $3 \leq d \leq 3 w$. Here $\delta_{k, l}$ denotes the Dirac-delta.

Proof. The total weight of tetragons after $(n-1)$ steps is $n$. The total weight of triangles after $(n-1)$ steps is $4 n$. The total weight of the triangles having a fixed common vertex of weight $w$ is $3 w$. Moreover, after $(n-1)$ steps, we have the following. When we choose three vertices randomly, then the probability that a given vertex is chosen is

$$
\frac{\left(\begin{array}{c}
V_{n-1}-1 \\
2
\end{array}\right)}{\left(\begin{array}{c}
V_{n-1} \\
3
\end{array}\right)}=\frac{3}{V_{n-1}} \text {. }
$$

When we choose four vertices randomly, then the probability that a given vertex is chosen is

$$
\frac{\left(\begin{array}{c}
V_{n-1}-1 \\
3
\end{array}\right)}{\left(\begin{array}{c}
V_{n-1} \\
4
\end{array}\right)}=\frac{4}{V_{n-1}}
$$

Therefore the probability that an old vertex of weight $w$ takes part in the interaction at step $n$ is

$$
\begin{gathered}
p\left(r \frac{3 w}{4 n}+(1-r) \frac{3}{V_{n-1}}\right)+(1-p)\left(q \frac{w}{n}+(1-q) \frac{4}{V_{n-1}}\right) \\
=\frac{w}{n} \alpha+\frac{p}{V_{n-1}} \beta,
\end{gathered}
$$

where $\alpha$ and $\beta$ are defined by (4). A new vertex always takes part in the interaction. At each step with probability $p$ a new vertex with weight 1 and with degree 3 is born. This explains term $p \delta_{d, 3} \delta_{w, 1}$ in (5).

Consider a fixed vertex with weight $w$ and degree $d$. The probability of the event that in the $n$th step

(i) neither its degree $d$ nor its weight $w$ change is

$$
1-\left(\frac{w}{n} \alpha+\frac{p}{V_{n-1}} \beta\right)
$$

(ii) its degree does not change but its weight is increased by 1 is

$$
(1-p)\left(q \frac{w-1}{n}+(1-q) \frac{\left(\begin{array}{c}
d \\
3
\end{array}\right)}{\left(\begin{array}{c}
\left.V_{n-1}\right) \\
4
\end{array}\right)}\right)
$$

(iii) both its degree and its weight are increased by 1 is

$$
\begin{aligned}
& p\left(r \frac{3(w-1)}{4 n}+(1-r) \frac{\left(\begin{array}{c}
d-1 \\
2
\end{array}\right)}{\left(\begin{array}{c}
V_{n-1} \\
3
\end{array}\right)}\right) \\
& +(1-p)(1-q) \frac{\left(\begin{array}{c}
d-1 \\
2
\end{array}\right)\left(V_{n-1}-d\right)}{\left(\begin{array}{c}
V_{n-1} \\
4
\end{array}\right)}
\end{aligned}
$$

(iv) its degree is increased by 2 and its weight is increased by 1 is

$$
\begin{aligned}
& p(1-r) \frac{(d-2)\left(V_{n-1}-d+1\right)}{\left(\begin{array}{c}
V_{n-1} \\
3
\end{array}\right)} \\
& +(1-p)(1-q) \frac{(d-2)\left(\begin{array}{c}
V_{n-1}-d+1 \\
2
\end{array}\right)}{\left(\begin{array}{c}
V_{n-1} \\
4
\end{array}\right)}
\end{aligned}
$$

(v) its degree is increased by 3 and its weight is increased by 1 is

$$
p(1-r) \frac{\left(\begin{array}{c}
V_{n-1}-d+2 \\
2
\end{array}\right)}{\left(\begin{array}{c}
V_{n-1} \\
3
\end{array}\right)}+(1-p)(1-q) \frac{\left(\begin{array}{c}
V_{n-1}-d+2 \\
3
\end{array}\right)}{\left(\begin{array}{c}
V_{n-1} \\
4
\end{array}\right)} .
$$

Using the above formulae, we obtain (5). [7].

The following theorem is an extension of Theorem 3.1 in

Theorem 3. Let $0<p<1, q>0, r>0$ and $(1-r)(1-q)>0$. For any fixed $w$ and $d$ with $1 \leq w$ and $3 \leq d \leq 3 w$ one has

$$
\frac{X(n, d, w)}{V_{n}} \longrightarrow x_{d, w}
$$

almost surely as $n \rightarrow \infty$, where $x_{d, w}$ are fixed nonnegative numbers. Furthermore, the numbers $x_{d, w}$ satisfy the following recurrence:

$$
\begin{gathered}
x_{3,1}=\frac{1}{\alpha+\beta+1}, \quad x_{d, 1}=0, \quad \text { for } d \neq 3 \\
x_{d, w}=\frac{1}{\alpha w+\beta+1}\left[\alpha_{1}(w-1) x_{d, w-1}+\alpha_{2}(w-1) x_{d-1, w-1}\right. \\
\left.+\beta x_{d-3, w-1}\right]
\end{gathered}
$$

for $w \geq 2,3 \leq d \leq 3 w$, where $\alpha_{1}, \alpha_{2}, \alpha$, and $\beta$ are given by (4). One has $x_{d, w}>0$ if $w \geq 1,3 \leq d \leq 3 w$ unless $d=3 w-1$. If $d=3 w-1$, then one has $x_{d, w}=0$; moreover, in this case

$$
\frac{X(n, d, w)}{V_{n}}=o\left(n^{-a}\right),
$$

where $a$ is a positive number which may depend on $w$ and $d$. If $3 \leq d \leq 3 w$ does not satisfied, then $x_{d, w}=0$. 
Proof. During the proof we take care of the case $x_{d, w}=0$ which does not appear in [7]. Introduce notation

$$
c(n, w)=\prod_{i=w-1}^{n}\left(1-\frac{\alpha w}{i}-\frac{\beta p}{V_{i-1}}\right)^{-1}, \quad n \geq w-1, w \geq 1 .
$$

$c(n, w)$ is an $\mathscr{F}_{n-1}$-measurable random variable. Applying the Marcinkiewicz strong law of large numbers to the number of vertices, we have

$$
V_{n}=p n+o\left(n^{1 / 2+\varepsilon}\right)
$$

almost surely, for any $\varepsilon>0$. obtain

Using (18) and the Taylor expansion for $\log (1+x)$, we

$$
\begin{aligned}
\log c(n, w) & =-\sum_{i=w-1}^{n} \log \left(1-\frac{\alpha w}{i}-\frac{\beta}{i+o\left(i^{1 / 2+\varepsilon}\right)}\right) \\
& =(\alpha w+\beta) \sum_{i=w-1}^{n} \frac{1}{i}+O(1),
\end{aligned}
$$

where the error term is convergent as $n \rightarrow \infty$. Therefore

$$
c(n, w) \sim a_{w} n^{\alpha w+\beta}
$$

almost surely, as $n \rightarrow \infty$, where $a_{w}$ is a positive random variable.

Let

$Z(n, d, w)=c(n, w) X(n, d, w) \quad$ for $1 \leq w, 3 \leq d \leq 3 w$.

Using (5), we can see that $\left\{Z(n, d, w), \mathscr{F}_{n}, n=w-1, w, w+\right.$ $1, \ldots\}$ is a nonnegative submartingale for any fixed $1 \leq w$, $3 \leq d \leq 3 w$. Define $Z(n, d, w)=0$ for $n=1,2, \ldots, w-2$. Applying the Doob-Meyer decomposition to $Z(n, d, w)$, we can write

$$
Z(n, d, w)=M(n, d, w)+A(n, d, w),
$$

where $M(n, d, w)$ is a martingale and $A(n, d, w)$ is a predictable increasing process. The general form of $M(n, d, w)$ and $A(n, d, w)$ is the following:

$$
\begin{aligned}
& M(n, d, w)=\sum_{i=1}^{n}\left[Z(i, d, w)-\mathbb{E}\left(Z(i, d, w) \mid \mathscr{F}_{i-1}\right)\right], \\
& A(n, d, w) \\
& =\mathbb{E} Z(1, d, w) \\
& \quad+\sum_{i=2}^{n}\left[\mathbb{E}\left(Z(i, d, w) \mid \mathscr{F}_{i-1}\right)-Z(i-1, d, w)\right],
\end{aligned}
$$

where $\mathscr{F}_{0}$ is the trivial $\sigma$-algebra. Using (5) and (24), we obtain

$$
\begin{aligned}
& A(n, d, w) \\
& =\mathbb{E} Z(1, d, w) \\
& +\sum_{i=2}^{n}[c(i, w) X(i-1, d, w-1)(1-p) \\
& \times\left(q \frac{w-1}{i}+(1-q) \frac{\left(\begin{array}{c}
d \\
3
\end{array}\right)}{\left(\begin{array}{c}
V_{i-1} \\
4
\end{array}\right)}\right) \\
& +c(i, w) X(i-1, d-1, w-1) \\
& \times\left(p\left(r \frac{3(w-1)}{4 i}+(1-r) \frac{\left(\begin{array}{c}
d-1 \\
2
\end{array}\right)}{\left(\begin{array}{c}
V_{i-1} \\
3
\end{array}\right)}\right)\right. \\
& \left.+(1-p)(1-q) \frac{\left(\begin{array}{c}
d-1 \\
2
\end{array}\right)\left(V_{i-1}-d\right)}{\left(\begin{array}{c}
V_{i-1} \\
4
\end{array}\right)}\right) \\
& +c(i, w) X(i-1, d-2, w-1) \\
& \times\left(p(1-r) \frac{(d-2)\left(V_{i-1}-d+1\right)}{\left(\begin{array}{c}
V_{i-1} \\
3
\end{array}\right)}\right. \\
& \left.+(1-p)(1-q) \frac{(d-2)\left(\begin{array}{c}
V_{i-1}-d+1 \\
2
\end{array}\right)}{\left(\begin{array}{c}
V_{i-1} \\
4
\end{array}\right)}\right) \\
& +c(i, w) X(i-1, d-3, w-1) \\
& \times\left(p(1-r) \frac{\left(\begin{array}{c}
V_{i-1}-d+2 \\
2
\end{array}\right)}{\left(\begin{array}{c}
V_{i-1} \\
3
\end{array}\right)}\right. \\
& \left.+(1-p)(1-q) \frac{\left(\begin{array}{c}
V_{i-1}-d+2 \\
3
\end{array}\right)}{\left(\begin{array}{c}
V_{i-1} \\
4
\end{array}\right)}\right) \\
& \left.+c(i, w) p \delta_{d, 3} \delta_{w, 1}\right] .
\end{aligned}
$$

Let $B(n, d, w)$ denote the sum of the conditional variances of the process $Z(n, d, w)$. Now we will give an upper bound of $B(n, d, w)$ as follows:

$$
\begin{aligned}
B & (n, d, w) \\
= & \sum_{i=2}^{n} \mathbb{D}^{2}\left(Z(i, d, w) \mid \mathscr{F}_{i-1}\right) \\
= & \sum_{i=2}^{n} \mathbb{E}\left\{\left(Z(i, d, w)-\mathbb{E}\left(Z(i, d, w) \mid \mathscr{F}_{i-1}\right)\right)^{2} \mid \mathscr{F}_{i-1}\right\} \\
= & \sum_{i=2}^{n} c(i, w)^{2} \\
& \quad \times \mathbb{E}\left\{\left(X(i, d, w)-\mathbb{E}\left(X(i, d, w) \mid \mathscr{F}_{i-1}\right)\right)^{2} \mid \mathscr{F}_{i-1}\right\}
\end{aligned}
$$




$$
\begin{aligned}
& \leq \sum_{i=2}^{n} c(i, w)^{2} \\
& \quad \times \mathbb{E}\left\{(X(i, d, w)-X(i-1, d, w))^{2} \mid \mathscr{F}_{i-1}\right\} \\
& \leq 16 \sum_{i=2}^{n} c(i, w)^{2}=O\left(n^{2(\alpha w+\beta)+1}\right) .
\end{aligned}
$$

Above we used that $c(i, w)$ is $\mathscr{F}_{i-1}$-measurable and at each step four vertices can interact. Jensen's inequality implies that $M^{2}(n, d, w)$ is a (nonnegative) submartingale if $M(n, d, w)$ is a martingale. Now we can apply the Doob-Meyer decomposition to $M^{2}(n, d, w)$. It is known that $B(n, d, w)$, that is the sum of the conditional variances of terms $Z(n, d, w)$ from formula (26), is the same (up to an additive constant) as the increasing predictable process in the Doob-Meyer decomposition of the nonnegative submartingale $M^{2}(n, d, w)$. Therefore the DoobMeyer decomposition is

$$
M^{2}(n, d, w)=Y(n, d, w)+B(n, d, w)
$$

where $Y(n, d, w)$ is a martingale and the predictable increasing process $B(n, d, w)$ is given by $(26)$.

We use induction on $w$. Let $w=1$. We can see that a vertex of weight 1 could take part in an interaction when it was born. Therefore its degree must be equal to 3. By (25) and (20),

$$
A(n, 3,1) \sim p \sum_{i=2}^{n} c(i, 1) \sim p \sum_{i=2}^{n} a_{1} i^{\alpha+\beta} \sim p a_{1} \frac{n^{\alpha+\beta+1}}{\alpha+\beta+1}
$$

almost surely as $n \rightarrow \infty$. By $(26), B(n, 3,1)=O\left(n^{2(\alpha+\beta)+1}\right)$ and therefore $(B(n, 3,1))^{1 / 2} \log B(n, 3,1)=O(A(n, 3,1))$. It follows from Proposition VII-2-4 of [8] (see Proposition 10 in the Appendix) that

$$
Z(n, 3,1) \sim A(n, 3,1)
$$$$
\text { a.s. on the event }\{A(n, 3,1) \longrightarrow \infty\} \text { as } n \longrightarrow \infty \text {. }
$$

As, by $(28), A(n, 3,1) \rightarrow \infty$ almost surely, therefore using (18) and (20), relation (29) implies

$$
\begin{aligned}
\frac{X(n, 3,1)}{V_{n}} & =\frac{Z(n, 3,1)}{c(n, 1) V_{n}} \sim \frac{A(n, 3,1)}{c(n, 1) V_{n}} \\
& \sim \frac{p a_{1}\left(n^{\alpha+\beta+1} /(\alpha+\beta+1)\right)}{a_{1} n^{\alpha+\beta} p n} \\
& =\frac{1}{\alpha+\beta+1}=x_{3,1}>0
\end{aligned}
$$

almost surely. So (14) is valid for $w=1$.

Now let $w=2$. In this case the degree of the vertex must be $3 \leq d \leq 6$. If $w=2$ and $d=3$, , or 6 , then we will show $x_{d, w}>0$. By (18), (20), and (25), we can find the asymptotic behaviour of $A(n, d, 2)$ as

$$
\begin{gathered}
A(n, 3,2) \sim p a_{2} \frac{n^{2 \alpha+\beta+1}}{2 \alpha+\beta+1}(1-p) q x_{3,1} \rightarrow \infty \\
A(n, 4,2) \sim p a_{2} \frac{n^{2 \alpha+\beta+1}}{2 \alpha+\beta+1} \frac{3}{4} p r x_{3,1} \rightarrow \infty, \\
A(n, 6,2) \sim p a_{2} \frac{n^{2 \alpha+\beta+1}}{2 \alpha+\beta+1} \\
\times\left[3(1-r)+\frac{4(1-p)(1-q)}{p}\right] x_{3,1} \rightarrow \infty .
\end{gathered}
$$

Moreover, by (26), $B(n, d, 2)=O\left(n^{2(2 \alpha+\beta)+1}\right)$; thus, $(B(n, d, 2))^{1 / 2} \log B(n, d, 2)=O(A(n, d, 2))$ for $d=3,4$, and 6 . Therefore in these cases $Z(n, d, 2) \sim A(n, d, 2)$ almost surely on $\{A(n, d, 2) \rightarrow \infty\}$ as $n \rightarrow \infty$. It implies that

$$
\begin{aligned}
\frac{X(n, d, 2)}{V_{n}} & =\frac{Z(n, d, 2)}{c(n, 2) V_{n}} \sim \frac{A(n, d, 2)}{c(n, 2) V_{n}} \\
& \sim \frac{p a_{2}\left(n^{2 \alpha+\beta+1} /(2 \alpha+\beta+1)\right)}{a_{2} n^{2 \alpha+\beta} p n} T_{d, 2} \\
& =\frac{T_{d, 2}}{2 \alpha+\beta+1}
\end{aligned}
$$

with appropriate $T_{d, 2}$. In particular, we have

$$
\begin{gathered}
\frac{X(n, 3,2)}{V_{n}} \longrightarrow \frac{(1-p) q x_{3,1}}{2 \alpha+\beta+1}=x_{3,2}>0, \\
\frac{X(n, 4,2)}{V_{n}} \longrightarrow \frac{(3 / 4) p r x_{3,1}}{2 \alpha+\beta+1}=x_{4,2}>0, \\
\frac{X(n, 6,2)}{V_{n}} \longrightarrow\left[3(1-r)+\frac{4(1-p)(1-q)}{p}\right] \frac{x_{3,1}}{2 \alpha+\beta+1} \\
=x_{6,2}>0,
\end{gathered}
$$

as $n \rightarrow \infty$. So (14) is valid for $w=2$ and $d=3,4,6$.

However, the case $d=5, w=2$ is different from the previous cases. As $x_{5,2}=0$, the relation $X(n, 5,2) / V_{n} \rightarrow x_{5,2}$ would not be enough to proceed the induction. By (25) and using Remark 1, we have

$$
\begin{aligned}
A(n, 5,2)= & \mathbb{E} Z(1,5,2) \\
& +\sum_{i=2}^{n}[c(i, 2) X(i-1,3,1)
\end{aligned}
$$




$$
\begin{aligned}
& \times\left(p(1-r) \frac{3\left(V_{i-1}-4\right)}{\left(\begin{array}{c}
V_{i-1} \\
3
\end{array}\right)}\right. \\
& \left.\left.\quad+(1-p)(1-q) \frac{3\left(\begin{array}{c}
V_{i-1}-4 \\
2
\end{array}\right)}{\left(\begin{array}{c}
V_{i-1} \\
4
\end{array}\right)}\right)\right] .
\end{aligned}
$$

Using (18), (34), and the limit of $X(i-1,3,1) / V_{i-1}$, we obtain

$$
\begin{aligned}
A(n, 5,2) \sim & \sum_{i=2}^{n} a_{2} i^{2 \alpha+\beta} x_{3,1} i p \\
& \times\left[\frac{18 p(1-r)}{(p i)^{2}}+\frac{36(1-p)(1-q)}{(p i)^{2}}\right] \\
& \sim a_{2} x_{3,1} \frac{18}{p} \sum_{i=2}^{n} i^{2 \alpha+\beta-1}(p(1-r)+2(1-p)(1-q)) \\
& \sim a_{2} x_{3,1} \frac{18}{p}(p(1-r)+2(1-p)(1-q)) \\
& \times \frac{n^{2 \alpha+\beta}}{2 \alpha+\beta} \longrightarrow \infty, \quad \text { as } n \longrightarrow \infty .
\end{aligned}
$$

Now we have

$$
\frac{X(n, 5,2)}{V_{n}}=\frac{Z(n, 5,2)}{c(n, 2) V_{n}}=\frac{M(n, 5,2)+A(n, 5,2)}{c(n, 2) V_{n}} .
$$

We denoted by $B(n, d, w)$ the increasing predictable process in the Doob-Meyer decomposition of $M^{2}(n, d, w)$. We know, by $(26)$, that $B(n, 5,2)=O\left(n^{4 \alpha+2 \beta+1}\right)$ and so $(B(n, 5,2))^{1 / 2} \log B(n, 5,2)=O\left(n^{2 \alpha+\beta+1 / 2+\varepsilon}\right)$ with arbitrary small positive $\varepsilon$.

Applying Propositions VII-2-3 and VII-2-4 of [8] (see Proposition 9 in the Appendix), we have

$$
\begin{aligned}
M(n, 5,2) & =o\left((B(n, 5,2))^{1 / 2} \log B(n, 5,2)\right) \\
& =o\left(n^{2 \alpha+\beta+1 / 2+\varepsilon}\right) \quad \text { a.e. on }\{B(n, 5,2) \rightarrow \infty\} .
\end{aligned}
$$

Moreover, on the set $\{B(\infty, 5,2)<\infty\}$, the sequence $M(n, 5,2)$ is almost surely. convergent. So $M(n, 5,2)=$ $o\left(n^{2 \alpha+\beta+1 / 2+\varepsilon}\right)$ almost surely. Therefore, using (18), (20), and (35), we obtain

$$
\begin{aligned}
\frac{X(n, 5,2)}{V_{n}} & =\frac{M(n, 5,2)+A(n, 5,2)}{c(n, 2) V_{n}} \leq C \frac{n^{2 \alpha+\beta+1 / 2+\varepsilon}}{n^{2 \alpha+\beta} n} \\
& =C \frac{1}{n^{a}} \longrightarrow 0
\end{aligned}
$$

if $n \rightarrow \infty$ where $1 / 4<a<1 / 2$. So the proposition is valid for $w=1$ and $w=2$.

Now, suppose that the statement is true for all weights less than $w$ and for all possible degrees. First we study the positive limits. Consider $A(n, d, w)$ in (25) and assume that at least one of the coefficients $x_{d, w-1}, x_{d-1, w-1}, x_{d-3, w-1}$ is positive. Then by (18), (20), and using the induction hypothesis, we see that

$$
\begin{aligned}
& A(n, d, w) \\
& \sim \sum_{i=2}^{n}\left[c(i, w) x_{d, w-1} p i(1-p) q \frac{w-1}{i}\right. \\
& +c(i, w) x_{d-1, w-1} \operatorname{pipr} \frac{3(w-1)}{4 i} \\
& +c(i, w) x_{d-3, w-1} p i \\
& \left.\times\left(\frac{3 p(1-r)}{p i}+\frac{4(1-p)(1-q)}{p i}\right)\right] \\
& \sim \sum_{i=2}^{n} a_{w} i^{\alpha w+\beta} \\
& \times\left[x_{d, w-1} p(1-p) q(w-1)\right. \\
& +x_{d-1, w-1} \frac{3}{4} p^{2} r(w-1) \\
& \left.+x_{d-3, w-1}(3 p(1-r)+4(1-p)(1-q))\right] \\
& \sim p a_{w} \frac{n^{\alpha w+\beta+1}}{\alpha w+\beta+1} \\
& \times\left[(1-p) q(w-1) x_{d, w-1}\right. \\
& +\frac{3}{4} \operatorname{pr}(w-1) x_{d-1, w-1} \\
& \left.+\left(3(1-r)+\frac{4(1-p)(1-q)}{p}\right) x_{d-3, w-1}\right] .
\end{aligned}
$$

In the above computation we deleted all terms having asymptotically smaller degree than the largest one.

Consider the case when $3 \leq d \leq 3 w$ and $d \neq 3 w-$ 1. In this case, by the induction hypothesis, there is at least one positive term in (39). Therefore (39) implies $A(n, d, w) \sim p a_{w} n^{\alpha w+\beta+1} x_{d, w} \rightarrow \infty$ (because $x_{d, w}>0$ ). In this case $(B(n, d, w))^{1 / 2} \log B(n, d, w)=O(A(n, d, w))$. So, using Proposition VII-2-4 of [8] (see Proposition 10 in the Appendix), we have $Z(n, d, w) \sim A(n, d, w)$. Therefore

$$
\begin{aligned}
\frac{X(n, d, w)}{V_{n}} & =\frac{Z(n, d, w)}{c(n, w) V_{n}} \sim \frac{A(n, d, w)}{c(n, w) V_{n}} \sim \frac{p a_{w} n^{\alpha w+\beta+1} x_{d, w}}{a_{w} n^{\alpha w+\beta} p n} \\
& =x_{d, w} \quad \text { a.s. as } n \longrightarrow \infty,
\end{aligned}
$$

where, by (39),

$$
\begin{aligned}
& x_{d, w} \\
& =\frac{1}{\alpha w+\beta+1} \\
& \quad \times\left[\alpha_{1}(w-1) x_{d, w-1}+\alpha_{2}(w-1) x_{d-1, w-1}+\beta x_{d-3, w-1}\right],
\end{aligned}
$$

with $\alpha_{1}, \alpha_{2}, \alpha$, and $\beta$ defined by (4). 
To handle the case when the limit is 0, we argue as follows. Consider the case when $d=3 w-1$. By (25) and using the induction hypothesis, we have

$$
\begin{aligned}
& A(n, 3 w-1, w) \\
& =\mathbb{E} Z(1,3 w-1, w) \\
& +\sum_{i=2}^{n}[c(i, w) X(i-1,3 w-3, w-1) \\
& \times\left(p(1-r) \frac{(3 w-3)\left(V_{i-1}-3 w+2\right)}{\left(\begin{array}{c}
V_{i-1} \\
3
\end{array}\right)}\right. \\
& \left.+(1-p)(1-q) \frac{(3 w-3)\left(\begin{array}{c}
V_{i-1}-3 w+2 \\
2
\end{array}\right)}{\left(\begin{array}{c}
V_{i-1} \\
4
\end{array}\right)}\right) \\
& +c(i, w) X(i-1,3 w-4, w-1) \\
& \times\left(p(1-r) \frac{\left(\begin{array}{c}
V_{i-1}-3 w+3 \\
2
\end{array}\right)}{\left(\begin{array}{c}
V_{i-1} \\
3
\end{array}\right)}\right. \\
& \left.\left.+(1-p)(1-q) \frac{\left(\begin{array}{c}
V_{i-1}-3 w+3 \\
3
\end{array}\right)}{\left(\begin{array}{c}
V_{i-1} \\
4
\end{array}\right)}\right)\right] \\
& \sim \sum_{i=2}^{n} a_{w} i^{\alpha w+\beta}\left[x_{3 w-3, w-1} C_{1} \frac{1}{p i}+O\left(\frac{1}{i^{a}}\right)\right] \\
& \leq C_{1} \frac{n^{\alpha w+\beta}}{\alpha w+\beta}+C_{2} \frac{n^{\alpha w+\beta+1-a}}{\alpha w+\beta+1-a} \\
& =O\left(n^{\alpha w+\beta+1-a}\right) \text {. }
\end{aligned}
$$

On the other hand, $B(n, d, w)=O\left(n^{2(\alpha w+\beta)+1}\right)$. Therefore, using (18), (20), and (42), by Propositions VII-2-3 and VII-24 of [8] (see Proposition 9 in the Appendix), we obtain

$$
\begin{aligned}
\frac{X(n, d, w)}{V_{n}} & =\frac{Z(n, d, w)}{c(n, w) V_{n}}=\frac{M(n, d, w)+A(n, d, w)}{c(n, w) V_{n}} \\
& =\frac{O\left(n^{\alpha w+\beta+1-a}\right)}{n^{\alpha w+\beta} p n}=O\left(n^{-a}\right) \longrightarrow 0 \\
& =x_{3 w-1, w}, \quad \text { a.s. }
\end{aligned}
$$

So we have obtained the desired result for the case of 0 limit as well.

\section{The Scale-Free Property for the Weights and Degrees}

Lemma 4. Let $p>0$ and define

$$
x_{w}=x_{3, w}+x_{4, w}+\cdots+x_{3 w, w}
$$

for $w=1,2, \ldots$. Then $x_{w}, w=1,2, \ldots$, are positive numbers satisfying the following recurrence:

$$
\begin{gathered}
x_{1}=\frac{1}{\alpha+\beta+1}, \\
x_{w}=\frac{\alpha(w-1)+\beta}{\alpha w+\beta+1} x_{w-1}, \quad \text { if } w>1,
\end{gathered}
$$

where $\alpha$ and $\beta$ are defined by (4). $x_{w}, w=1,2, \ldots$, is a discrete probability distribution. Moreover, $x_{d, w}, d=3,4, \ldots, 3 w, w=$ $1,2, \ldots$, is a two-dimensional discrete probability distribution.

Proof. If $\alpha=0$, then the statement is obvious. Now assume $\alpha \neq 0$. As $x_{d, w}$ is defined as $x_{d, w}=0$ for $d \notin\{3,4, \ldots, 3 w\}$, therefore $x_{w}=\sum_{d} x_{d, w}$. From the recurrence (15) for $x_{d, w}$, we obtain

$$
\begin{gathered}
x_{w}=\sum_{d=3}^{3 w} x_{d, w}=\sum_{d} x_{d, w} \\
=\frac{1}{\alpha w+\beta+1}\left[\alpha_{1}(w-1) \sum_{d} x_{d, w-1}\right. \\
+\alpha_{2}(w-1) \sum_{d} x_{d-1, w-1} \\
\left.+\beta \sum_{d} x_{d-3, w-1}\right] \\
=\frac{\alpha(w-1)+\beta}{\alpha w+\beta+1} x_{w-1} .
\end{gathered}
$$

Using this recursive formula for $x_{w}$, we obtain

$$
\begin{aligned}
x_{w} & =x_{1} \prod_{j=2}^{w} \frac{\alpha(j-1)+\beta}{\alpha j+\beta+1} \\
& =\frac{1}{\alpha+\beta+1} \frac{\alpha+\beta}{2 \alpha+\beta+1} \frac{2 \alpha+\beta}{3 \alpha+\beta+1} \cdots \frac{(w-1) \alpha+\beta}{w \alpha+\beta+1} \\
& =\frac{1}{\alpha w+\beta+1} \prod_{j=1}^{w-1} \frac{\beta / \alpha+j}{(\beta+1) / \alpha+j} \\
& =\frac{1}{\alpha w+\beta+1} \frac{\Gamma(w+\beta / \alpha)}{\Gamma(1+\beta / \alpha)} \frac{\Gamma(1+(\beta+1) / \alpha)}{\Gamma(w+(\beta+1) / \alpha)} \\
& =\frac{\Gamma(1+(\beta+1) / \alpha)}{\alpha \Gamma(1+\beta / \alpha)} \frac{\Gamma(w+\beta / \alpha)}{\Gamma(w+(\beta+1) / \alpha+1)} .
\end{aligned}
$$

Moreover, by [9], we have the following formula:

$$
\sum_{k=0}^{n} \frac{\Gamma(k+a)}{\Gamma(k+b)}=\frac{1}{a-b+1}\left[\frac{\Gamma(n+a+1)}{\Gamma(n+b)}-\frac{\Gamma(a)}{\Gamma(b-1)}\right] .
$$

Therefore, by some calculation, we obtain $\sum_{w=1}^{n} x_{w} \rightarrow 1$, as $n \rightarrow \infty$. So $\sum_{w=1}^{\infty} x_{w}=1$. As $\sum_{d} x_{d, w}=x_{w}$, so 
$\sum_{w=1}^{\infty} \sum_{d=3}^{3 w} x_{d, w}=1$ and therefore $x_{d, w}, d=3,4, \ldots, 3 w, w=$ $1,2, \ldots$, is a (proper) two-dimensional discrete probability distribution.

Let $X(n, w)$ denote the number of vertices of weight $w$ after $n$ steps. Next theorem is the scale-free property for the weights. It is an extension of Theorem 3.1 in [6].

Theorem 5. Let $0<p<1, q>0, r>0$ and $(1-r)(1-q)>0$. Then for all $w=1,2, \ldots$ one has

$$
\frac{X(n, w)}{V_{n}} \longrightarrow x_{w}=x_{3, w}+x_{4, w}+\cdots+x_{3 w, w}
$$

almost surely, as $n \rightarrow \infty$, where $x_{w}, w=1,2, \ldots$, are positive numbers satisfying the recurrence (45). Moreover,

$$
x_{w} \sim C w^{-(1+1 / \alpha)},
$$

as $w \rightarrow \infty$, with $C=\Gamma(1+(\beta+1) / \alpha) /(\alpha \Gamma(1+\beta / \alpha))$.

Proof. We have

$$
X(n, w)=X(n, 3, w)+X(n, 4, w)+\cdots+X(n, 3 w, w) .
$$

Therefore, by Theorem 3,

$$
\frac{X(n, w)}{V_{n}} \longrightarrow x_{3, w}+\cdots+x_{3 w, w}=x_{w}
$$

almost surely, as $n \rightarrow \infty$. Here each $x_{w}$ is positive.

Using formula (47) and the Stirling-formula for the Gamma function, we obtain

$$
\begin{aligned}
x_{w}= & \frac{\Gamma(1+(\beta+1) / \alpha)}{\alpha \Gamma(1+\beta / \alpha)} \frac{\Gamma(w+\beta / \alpha)}{\Gamma(w+(\beta+1) / \alpha+1)} \\
\sim & C_{0} \frac{(w+\beta / \alpha)^{(w+\beta / \alpha)}}{(w+\beta / \alpha+1 / \alpha+1)^{(w+\beta / \alpha+1 / \alpha+1)}} \\
= & C_{0}\left(\frac{(w+\beta / \alpha)}{(w+\beta / \alpha+1 / \alpha+1)}\right)^{(w+\beta / \alpha)} \\
& \times \frac{1}{(w+\beta / \alpha+1 / \alpha+1)^{1 / \alpha+1}} \sim C w^{-(1+1 / \alpha)},
\end{aligned}
$$

where $C_{0}=\Gamma(1+(\beta+1) / \alpha) /(\alpha \Gamma(1+\beta / \alpha))\left(1 /(1 / e)^{1+1 / \alpha}\right)$ and $C=\Gamma(1+(\beta+1) / \alpha)(\alpha \Gamma(1+\beta / \alpha))$.

Now, following [7], we construct a representation of the limiting joint distribution of degrees and weights.

Let $W$ be a random variable with distribution $\mathbb{P}(W=$ $w)=x_{w}, w=1,2, \ldots$. Let $\xi_{1} \equiv 3$ and $\xi_{2}, \xi_{3}, \ldots$ be independent random variables being independent of $W$, too. For $w \geq 2$ let $\xi_{w}$ have the following distribution:

$$
\begin{aligned}
& \mathbb{P}\left(\xi_{w}=0\right)=\frac{\alpha_{1}(w-1)}{\alpha(w-1)+\beta}, \\
& \mathbb{P}\left(\xi_{w}=1\right)=\frac{\alpha_{2}(w-1)}{\alpha(w-1)+\beta}, \\
& \mathbb{P}\left(\xi_{w}=3\right)=\frac{\beta}{\alpha(w-1)+\beta} .
\end{aligned}
$$

Introduce notation $S_{w}=\xi_{1}+\xi_{2}+\cdots+\xi_{w}$.

The following representation of the joint distribution of degrees and weights is useful to obtain scale-free property for degrees.

Theorem 6. $\mathbb{P}\left(S_{W}=d, W=w\right)=x_{d, w}$ for all $w=1,2, \ldots$, $d=3,4, \ldots, 3 w$.

Proof. It is easy to see that if $w=1$ and $d=3$, then we have

$$
\begin{aligned}
\mathbb{P}\left(S_{W}=3, W=1\right) & =\mathbb{P}\left(S_{1}=3, W=1\right) \\
& =\mathbb{P}\left(\xi_{1}=3, W=1\right) \\
& =\mathbb{P}(W=1)=x_{1}=x_{3,1} .
\end{aligned}
$$

If $w=2$ and $d=5$ we have

$$
\begin{aligned}
\mathbb{P}\left(S_{W}=5, W=2\right) & =\mathbb{P}\left(S_{2}=5, W=2\right) \\
& =\mathbb{P}\left(\xi_{1}=3, \xi_{2}=2, W=2\right) \\
& =0=x_{5,2} .
\end{aligned}
$$

Generally, for all $w=1,2, \ldots$ and $d=3 w-1$ we have

$$
\begin{aligned}
\mathbb{P}\left(S_{W}\right. & =3 w-1, W=w) \\
& =\mathbb{P}\left(\xi_{1}=3, \xi_{2}=k_{2}, \ldots, \xi_{w}=k_{w}, W=w\right) \\
& =0=x_{3 w-1, w},
\end{aligned}
$$

because one of $k_{2}, \ldots, k_{w}$ should be equal to 2 , which is of zero probability. Furthermore, using the recursion (45) and the assumption that $\xi_{i}$ are independent random variables which are independent of $W$, we have

$$
\begin{aligned}
\mathbb{P}\left(S_{W}=\right. & d, W=w) \\
= & \mathbb{P}\left(S_{w}=d, W=w\right) \\
= & \mathbb{P}\left(S_{w}=d\right) \mathbb{P}(W=w) \\
= & {\left[\mathbb{P}\left(S_{w-1}=d\right) \mathbb{P}\left(\xi_{w}=0\right)\right.} \\
& \quad+\mathbb{P}\left(S_{w-1}=d-1\right) \mathbb{P}\left(\xi_{w}=1\right) \\
& \left.+\mathbb{P}\left(S_{w-1}=d-3\right) \mathbb{P}\left(\xi_{w}=3\right)\right] \\
& \times \mathbb{P}(W=w-1) \frac{x_{w}}{x_{w-1}}
\end{aligned}
$$




$$
\begin{aligned}
&= {\left[\mathbb{P}\left(S_{w-1}=d\right) \mathbb{P}(W=w-1) \alpha_{1}(w-1)\right.} \\
&+\mathbb{P}\left(S_{w-1}=d-1\right) \mathbb{P}(W=w-1) \alpha_{2}(w-1) \\
&\left.+\mathbb{P}\left(S_{w-1}=d-3\right) \mathbb{P}(W=w-1) \beta\right] \\
& \times \frac{1}{\alpha w+\beta+1} \\
&= \frac{1}{\alpha w+\beta+1} \\
& \quad \times\left[\alpha_{1}(w-1) \mathbb{P}\left(S_{W}=d, W=w-1\right)\right. \\
& \quad+\alpha_{2}(w-1)\left(S_{W}=d-1, W=w-1\right) \\
&\left.\quad+\beta \mathbb{P}\left(S_{W}=d-3, W=w-1\right)\right] .
\end{aligned}
$$

Therefore, the sequence $\mathbb{P}\left(S_{W}=d, W=w\right)$ satisfies the same recursion (15) as $x_{d, w}$.

Theorem 7. Suppose that $\alpha_{1}>0$ and $\alpha_{2}>0$. Then

$$
\begin{aligned}
& x_{d, w}= x_{w} \frac{\alpha}{\sqrt{2 \pi \alpha_{1} \alpha_{2} w}} \\
& \times\left[\exp \left(-\frac{\left(d-\mathbb{E} S_{w}\right)^{2}}{2 \mathbb{D}^{2} S_{w}}\right)+O\left(w^{-1 / 2}\right)\right], \\
& \text { as } w \longrightarrow \infty,
\end{aligned}
$$

where the error term $O\left(w^{-1 / 2}\right)$ does not depend on $d$.

Proof. We can follow the ideas of Theorem 4.2 in [7]. For $w \geq$ 1 we have

$$
\begin{aligned}
\mathbb{E} \xi_{w} & =\frac{\alpha_{2}(w-1)}{\alpha(w-1)+\beta}+3 \frac{\beta}{\alpha(w-1)+\beta} \\
& =\frac{\alpha_{2}}{\alpha}+\frac{\left(3 \alpha-\alpha_{2}\right) \beta}{\alpha(\alpha(w-1)+\beta)} ;
\end{aligned}
$$

hence,

$$
\mathbb{E} S_{w}=\mathbb{E} \xi_{1}+\cdots+\mathbb{E} \xi_{w}=w \frac{\alpha_{2}}{\alpha}+\mathrm{O}(\log w),
$$

as $w \rightarrow \infty$. Similarly, by simple computation, we have

$$
\begin{gathered}
\mathbb{D}^{2} \xi_{w}=\frac{\alpha_{1} \alpha_{2}}{\alpha^{2}}+O\left(\frac{1}{w}\right), \\
\mathbb{D}^{2} S_{w}=\frac{\alpha_{1} \alpha_{2}}{\alpha^{2}} w+O(\log w),
\end{gathered}
$$

as $w \rightarrow \infty$.

Now, we can apply Theorem VII.2.5 in [10] (see Proposition 11 in the Appendix) for $S_{w}$. The conditions of that theorem are satisfied; therefore, we have

$$
\begin{aligned}
\sup _{d \in \mathbb{Z}}\left|\mathbb{D} S_{w} \mathbb{P}\left(S_{w}=d\right)-\frac{1}{\sqrt{2 \pi}} \exp \left(-\frac{\left(d-\mathbb{E} S_{w}\right)^{2}}{2 \mathbb{D}^{2} S_{w}}\right)\right| \\
=O\left(\frac{1}{\sqrt{w}}\right) .
\end{aligned}
$$

Using (62) and (63), we obtain $\left|\mathbb{D} S_{w}-\sqrt{\alpha_{1} \alpha_{2} w} / \alpha\right| \mathbb{P}\left(S_{w}=d\right)=$ $O\left(w^{-1 / 2}\right)$. Therefore, it follows from (63) that

$$
\begin{gathered}
\sup _{d \in \mathbb{Z}}\left|\frac{\sqrt{\alpha_{1} \alpha_{2} w}}{\alpha} \mathbb{P}\left(S_{w}=d\right)-\frac{1}{\sqrt{2 \pi}} \exp \left(-\frac{\left(d-\mathbb{E} S_{w}\right)^{2}}{2 \mathbb{D}^{2} S_{w}}\right)\right| \\
=O\left(\frac{1}{\sqrt{w}}\right) .
\end{gathered}
$$

The independence of $W$ and $\xi_{i}$ implies that $x_{d, w}=\mathbb{P}\left(S_{W}=\right.$ $d, W=w)=\mathbb{P}\left(S_{w}=d\right) x_{w}$. Using this in (64), we can obtain the desired result.

Our last theorem is an extension of Theorem 4.3 in [7] to the case of 4 interactions. The theorem shows the scale-free property for the degrees.

Theorem 8. Let $0<p<1, q>0, r>0$ and $(1-r)(1-q)>0$. Let us denote by $U(n, d)$ the number of vertices of degree $d$ after $n$ steps; that is, $U(n, d)=\sum_{w: d / 3 \leq w \leq n+1} X(n, d, w)$. Then, for any $d \geq 3$ one has

$$
\frac{U(n, d)}{V_{n}} \longrightarrow u_{d}=\sum_{w} x_{d, w}
$$

almost surely as $n \rightarrow \infty$, where $u_{d}$ are positive numbers. Furthermore,

$$
u_{d} \sim \frac{\Gamma(1+(\beta+1) / \alpha)}{\alpha_{2} \Gamma(1+\beta / \alpha)}\left(\frac{\alpha d}{\alpha_{2}}\right)^{-(1+1 / \alpha)}
$$

as $d \rightarrow \infty$.

Proof. By Theorems 3 and $6, X(n, d, w) / V_{n}$ converges almost surely to the distribution $x_{d, w}=\mathbb{P}\left(S_{W}=d, W=w\right)$. But the cardinalities of terms in $\sum_{w: d / 3 \leq w \leq n+1} X(n, d, w)$ are not bounded when $n \rightarrow \infty$. However, using that $x_{d, w}, d=$ $3,4, \ldots, 3 w, w=1,2, \ldots$ is a proper two-dimensional discrete distribution, the convergence of the marginal distributions is a consequence of the convergence of the two-dimensional distributions. So we obtain (65).

To obtain (66), we can apply the method of Theorem 4.3 in [7]. Let

$$
\begin{gathered}
f=\frac{\alpha}{\alpha_{2}} d, \\
H=H_{d}=\left\{w: f-f^{1 / 2+\varepsilon} \leq w \leq f+f^{1 / 2+\varepsilon}\right\}, \\
H^{-}=H_{d}^{-}=\left\{w: w<f-f^{1 / 2+\varepsilon}\right\}, \\
H^{+}=H_{d}^{+}=\left\{w: w>f+f^{1 / 2+\varepsilon}\right\}
\end{gathered}
$$

with some fixed $0<\varepsilon<1 / 6$. 
Using (61) and Hoeffding's exponential inequality (Theorem 2 in [11], see Proposition 12 in the Appendix) we obtain for $w \in H^{-}$

$$
\begin{aligned}
\mathbb{P}\left(S_{w}=d\right) & \leq \mathbb{P}\left(S_{w} \geq d\right) \\
& \leq \mathbb{P}\left(S_{w}-\mathbb{E} S_{w} \geq d-\frac{\alpha_{2}}{\alpha} w-O(\log w)\right) \\
& \leq \exp \left\{-\frac{2}{9 w}\left(d-\frac{\alpha_{2}}{\alpha} w-O(\log w)\right)^{2}\right\} \\
& =\exp \left\{-\frac{2}{9}\left(\frac{\alpha_{2}}{\alpha}\right)^{2} \frac{(f-w-O(\log w))^{2}}{w}\right\} .
\end{aligned}
$$

Here $w \in H^{-}$implies that

$$
\begin{aligned}
(f-w & -O(\log w))^{2} \\
& =(f-w)^{2}-2(f-w) O(\log w)+(O(\log w))^{2} \\
& \geq f^{1+2 \varepsilon}-O(f \log f) .
\end{aligned}
$$

Therefore in the case when $w \in H^{-}$we have

$$
\begin{aligned}
\mathbb{P}\left(S_{w}=d\right) & \leq \exp \left\{-\frac{2}{9}\left(\frac{\alpha_{2}}{\alpha}\right)^{2} \frac{f^{1+2 \varepsilon}-O(f \log f)}{f}\right\} \\
& =\exp \left\{-\frac{2}{9}\left(\frac{\alpha_{2}}{\alpha}\right)^{2} f^{2 \varepsilon}+O(\log f)\right\} .
\end{aligned}
$$

Using this, we can obtain that

$$
\begin{aligned}
\mathbb{P}\left(S_{W}\right. & \left.=d, W \in H^{-}\right) \\
& =\sum_{w \in H^{-}} \mathbb{P}\left(S_{w}=d, W=w\right) \\
& \leq \sum_{w \in H^{-}} \mathbb{P}\left(S_{w}=d\right) \\
& \leq f \exp \left\{-\frac{2}{9}\left(\frac{\alpha_{2}}{\alpha}\right)^{2} f^{2 \varepsilon}+O(\log f)\right\} \\
& =o\left(f^{-(1+1 / \alpha)}\right) .
\end{aligned}
$$

Similarly, if $w \in H^{+}$, again by Hoeffding's inequality, we have

$$
\begin{aligned}
\mathbb{P}\left(S_{w}=d\right) & \leq \mathbb{P}\left(S_{w} \leq d\right) \\
& \leq \mathbb{P}\left(S_{w}-\mathbb{E} S_{w} \leq d-\frac{\alpha_{2}}{\alpha} w\right) \\
& \leq \exp \left\{-\frac{2}{9 w}\left(d-\frac{\alpha_{2}}{\alpha} w\right)^{2}\right\} \\
& =\exp \left\{-\frac{2}{9}\left(\frac{\alpha_{2}}{\alpha}\right)^{2} \frac{(f-w)^{2}}{w}\right\} .
\end{aligned}
$$

Using that $w \in H^{+}$and $1 / 2+\varepsilon<1$, we obtain $2(w-f) \geq$ $f^{1 / 2+\varepsilon}+w-f \geq f^{1 / 2+\varepsilon}+(w-f)^{1 / 2+\varepsilon} \geq w^{1 / 2+\varepsilon}$. Therefore

$$
\begin{aligned}
\mathbb{P}\left(S_{w}=d\right) & \leq \exp \left\{-\frac{2}{9}\left(\frac{\alpha_{2}}{\alpha}\right)^{2} \frac{w^{1+2 \varepsilon}}{4 w}\right\} \\
& =\exp \left\{-\frac{1}{18}\left(\frac{\alpha_{2}}{\alpha}\right)^{2} w^{2 \varepsilon}\right\} .
\end{aligned}
$$

Hence

$$
\begin{aligned}
\mathbb{P}\left(S_{W}=d, W \in H^{+}\right) & \leq \sum_{\{w: f<w\}} \exp \left\{-\frac{1}{18}\left(\frac{\alpha_{2}}{\alpha}\right)^{2} w^{2 \varepsilon}\right\} \\
& =o\left(f^{-(1+1 / \alpha)}\right) .
\end{aligned}
$$

Now consider the case when $w \in H=H_{d}$. First we need some general facts. Consider the set

$$
B=\left\{(d, w): w \geq 1, d \geq 3, w \in H_{d}\right\} .
$$

It is easy to see that when $(d, w) \in B$ then $d \rightarrow \infty$ if and only if $w \rightarrow \infty$. More precisely,

$$
\frac{w}{d} \longrightarrow 1, \quad \text { if } d \longrightarrow \infty,(d, w) \in B
$$

As $w \in H$, so we have $w=f+O\left(f^{1 / 2+\varepsilon}\right)$. Then (with $\varepsilon_{1}>0$ arbitrarily small)

$$
\begin{aligned}
-\frac{\left(d-\mathbb{E} S_{w}\right)^{2}}{2 \mathbb{D}^{2} S_{w}}= & -\frac{\left(d-w\left(\alpha_{2} / \alpha\right)-O(\log w)\right)^{2}}{2\left(\alpha_{1} \alpha_{2} / \alpha^{2}\right) w+O(\log w)} \\
= & -\frac{\alpha_{2}}{\alpha_{1}} \frac{(f-w-O(\log w))^{2}}{2 w+O(\log w)} \\
= & -\frac{\alpha_{2}}{\alpha_{1}} \frac{(f-w)^{2}+O\left(f^{1 / 2+\varepsilon+\varepsilon_{1}}\right)}{2 w+O(\log w)} \\
= & -\frac{\alpha_{2}}{\alpha_{1}} \frac{(f-w)^{2}+O\left(f^{1 / 2+\varepsilon+\varepsilon_{1}}\right)}{2 f} \\
& \times \frac{2 f}{2 f+O\left(f^{1 / 2+\varepsilon}\right)} \\
= & -\frac{\alpha_{2}}{\alpha_{1}} \frac{(f-w)^{2}+O\left(f^{1 / 2+\varepsilon+\varepsilon_{1}}\right)}{2 f} \\
& \times\left[1-\frac{O\left(f^{1 / 2+\varepsilon}\right)}{2 f+O\left(f^{1 / 2+\varepsilon}\right)}\right] \\
= & -\frac{\alpha_{2}}{\alpha_{1}} \frac{(f-w)^{2}}{2 f}+O\left(f^{-1 / 2+3 \varepsilon}\right),
\end{aligned}
$$

as $d \rightarrow \infty$. Here the error term does not depend on $w$. We will apply Theorem 7 , that is, formula (59). The asymptotic 
behaviour of $x_{w}$ is known from (50). Using these facts and (77), we obtain

$$
\begin{aligned}
& x_{d, w} \\
& \sim C w^{-(1+1 / \alpha)} \frac{\alpha}{\sqrt{2 \pi \alpha_{1} \alpha_{2} w}} \\
& \quad \times\left[\exp \left\{-\frac{\alpha_{2}}{\alpha_{1}} \frac{(f-w)^{2}}{2 f}+O\left(f^{-1 / 2+3 \varepsilon}\right)\right\}+O\left(w^{-1 / 2}\right)\right] \\
& \sim C f^{-(1+1 / \alpha)} \frac{\alpha}{\alpha_{2}} \frac{1}{\sqrt{2 \pi\left(\alpha_{1} / \alpha_{2}\right) f}} \exp \left\{-\frac{(f-w)^{2}}{2\left(\alpha_{1} / \alpha_{2}\right) f}\right\}
\end{aligned}
$$

as $d \rightarrow \infty$ and $w \in H$, where $C=\Gamma(1+(\beta+1) / \alpha) /(\alpha \Gamma(1+$ $\beta / \alpha))$. Therefore

$$
\begin{aligned}
& \sum_{w \in H} x_{d, w} \\
& \sim \sum_{f-f^{1 / 2+\varepsilon}<w<f+f^{1 / 2+\varepsilon}} C f^{-(1+1 / \alpha)} \frac{\alpha}{\alpha_{2}} \frac{1}{\sqrt{2 \pi\left(\alpha_{1} / \alpha_{2}\right) f}} \\
& \times \exp \left\{-\frac{(f-w)^{2}}{2\left(\alpha_{1} / \alpha_{2}\right) f}\right\} \\
& =C f^{-(1+1 / \alpha)} \frac{\alpha}{\alpha_{2}} \sum_{-f^{1 / 2+\varepsilon}<k<f^{1 / 2+\varepsilon}} \frac{1}{\sqrt{2 \pi\left(\alpha_{1} / \alpha_{2}\right) f}} \\
& \times \exp \left\{-\frac{k^{2}}{2\left(\alpha_{1} / \alpha_{2}\right) f}\right\} \\
& =A \sum_{-f^{\varepsilon}<k / \sqrt{f}<f^{\varepsilon}} \frac{1}{\sqrt{f}} \frac{1}{\sqrt{2 \pi\left(\alpha_{1} / \alpha_{2}\right)}} \\
& \times \exp \left\{-\frac{(k / \sqrt{f})^{2}}{2\left(\alpha_{1} / \alpha_{2}\right)}\right\} \\
& \longrightarrow A \int_{-\infty}^{+\infty} \frac{1}{\sqrt{2 \pi\left(\alpha_{1} / \alpha_{2}\right)}} \exp \left\{-\frac{x^{2}}{2\left(\alpha_{1} / \alpha_{2}\right)}\right\} d x=A
\end{aligned}
$$

Thus we have

$$
\begin{aligned}
\mathbb{P}\left(S_{W}\right. & =d, W \in H) \sim A \\
& =\frac{\Gamma(1+(\beta+1) / \alpha)}{\alpha_{2} \Gamma(1+\beta / \alpha)}\left(\frac{\alpha d}{\alpha_{2}}\right)^{-(1+1 / \alpha)},
\end{aligned}
$$

as $d \rightarrow \infty$. Finally, (71), (74), and (80) imply

$$
\begin{aligned}
u_{d}= & \sum_{w} x_{d, w} \\
= & \sum_{w \in H^{-}} x_{d, w}+\sum_{w \in H} x_{d, w} \\
& +\sum_{w \in H^{+}} x_{d, w} \sim o\left(f^{-(1+1 / \alpha)}\right) \\
& +C \frac{\alpha}{\alpha_{2}} f^{-(1+1 / \alpha)}+o\left(f^{-(1+1 / \alpha)}\right) \\
\sim & \frac{\Gamma(1+(\beta+1) / \alpha)}{\alpha_{2} \Gamma(1+\beta / \alpha)}\left(\left(\alpha / \alpha_{2}\right) d\right)^{-(1+1 / \alpha)},
\end{aligned}
$$

as $d \rightarrow \infty$. The proof is complete.

\section{Appendix}

We use the following results on discrete time martingales. Let $\left\{Z_{n}, \mathscr{F}_{n}\right\}$ be a submartingale. Its Doob-Meyer decomposition is $Z_{n}=M_{n}+A_{n}$, where $\left\{M_{n}, \mathscr{F}_{n}\right\}$ is a martingale and $\left\{A_{n}, \mathscr{F}_{n}\right\}$ is an increasing predictable process. Here, up to an additive constant,

$$
A_{n}=\mathbb{E} Z_{1}+\sum_{i=2}^{n}\left(\mathbb{E}\left(Z_{i} \mid \mathscr{F}_{i-1}\right)-Z_{i-1}\right) .
$$

Now, $\left\{M_{n}^{2}, \mathscr{F}_{n}\right\}$ is again a submartingale. Let

$$
M_{n}^{2}=Y_{n}+B_{n}
$$

be the Doob-Meyer decomposition of $M_{n}^{2}$. Here, up to an additive constant,

$$
\begin{aligned}
B_{n} & =\sum_{i=2}^{n} \mathbb{D}^{2}\left(Z_{i} \mid \mathscr{F}_{i-1}\right) \\
& =\sum_{i=2}^{n} \mathbb{E}\left\{\left(Z_{i}-\mathbb{E}\left(Z_{i} \mid \mathscr{F}_{i-1}\right)\right)^{2} \mid \mathscr{F}_{i-1}\right\} .
\end{aligned}
$$

Proposition 9 (Propositions VII-2-3 and VII-2-4 of [8]). Let $M_{1}=0$. On the set $\left\{B_{\infty}<\infty\right\}$ the martingale $M_{n}$ almost surely converges to a finite limit. Moreover, $M_{n}=o\left(B_{n}^{1 / 2} \log B_{n}\right)$ almost surely on the set $\left\{B_{n} \rightarrow \infty\right\}$.

A consequence of the above proposition is the following.

Proposition 10 (Proposition 2.3 of [12]). Let $\left\{Z_{n}, \mathscr{F}_{n}\right\}$ be a square integrable nonnegative submartingale. If $B_{n}^{1 / 2} \log B_{n}=$ $O\left(A_{n}\right)$, then $Z_{n} \sim A_{n}$, as $n \rightarrow \infty$, almost surely on the set $\left\{A_{n} \rightarrow \infty\right\}$

We need the following local limit theorem. Let $X_{1}, X_{2}, \ldots$ be independent, integer valued random variables. Let $p_{j, m}=$ $\mathbb{P}\left(X_{j}=m\right)$ be the distribution, while $p_{j, m_{j}}=\max _{m} p_{j, m}$ be the maximal value of the distribution. Let $S_{n}=\sum_{i=1}^{n} X_{i}$ be the 
partial sum, $P_{n}(N)=\mathbb{P}\left(S_{n}=N\right)$ be its distribution, $M_{n}=$ $\sum_{i=1}^{n} \mathbb{E} X_{i}$ be the expectation, and $B_{n}=\sum_{i=1}^{n} \mathbb{E}\left(X_{i}-\mathbb{E} X_{i}\right)^{2}$ be the variance of $S_{n}$.

Proposition 11 (Theorem 5 and its consequence in Section VII, 2 of [10]). Assume that the greatest common divisor of the values

$$
\left\{m: \frac{1}{\log n} \sum_{j=1}^{n} p_{j, m_{j}} p_{j, m+m_{j}} \longrightarrow \infty\right\}
$$

is equal to 1; moreover,

$$
\begin{gathered}
\liminf \frac{B_{n}}{n}>0, \\
\limsup \frac{1}{n} \sum_{i=1}^{n} \mathbb{E}\left|X_{i}-\mathbb{E} X_{i}\right|^{3}<\infty .
\end{gathered}
$$

Then

$$
\sup _{N}\left|\sqrt{B_{n}} P_{n}(N)-\frac{1}{\sqrt{2 \pi}} \exp \left(\frac{\left(N-M_{n}\right)^{2}}{2 B_{n}}\right)\right|=O\left(\frac{1}{\sqrt{n}}\right) .
$$

The well-known Hoeffding's inequality is the following.

Proposition 12 (Theorem 2 of [11]). Let $X_{1}, X_{2}, \ldots, X_{n}$ be independent random variables, $a_{i} \leq X_{i} \leq b_{i}(i=1,2, \ldots, n)$. Let $\bar{X}=\left(X_{1}+X_{2}+\cdots+X_{n}\right) / n, \mu=\mathbb{E} \bar{X}$. Then for any $t>0$

$$
\mathbb{P}(\bar{X}-\mu \geq t) \leq \exp \left(\frac{-2 n^{2} t^{2}}{\sum_{i=1}^{n}\left(b_{i}-a_{i}\right)^{2}}\right) \text {. }
$$

\section{Acknowledgments}

The publication was supported by the TÁMOP-4.2.2.C$11 / 1 / \mathrm{KONV}-2012-0001$ project. The project has been supported by the European Union, cofinanced by the European Social Fund.

\section{References}

[1] R. Durrett, Random Graph Dynamics, Cambridge University Press, Cambridge, UK, 2007.

[2] A. L. Barabási and R. Albert, "Emergence of scaling in random networks," Science, vol. 286, no. 5439, pp. 509-512, 1999.

[3] B. Bollobás, O. Riordan, J. Spencer, and G. Tusnády, "The degree sequence of a scale-free random graph process," Random Structures \& Algorithms, vol. 18, no. 3, pp. 279-290, 2001.

[4] C. Cooper and A. Frieze, "A general model of web graphs," Random Structures \& Algorithms, vol. 22, no. 3, pp. 311-335, 2003.

[5] A. Sridharan, Y. Gao, K. Wu, and J. Nastos, "Statistical behavior of embeddedness and communities of overlapping cliques in online social networks," http://arxiv.org/abs/1009.1686.

[6] Á. Backhausz and T. F. Móri, "A random graph model based on 3-interactions," Annales Universitatis Scientiarum Budapestinensis de Rolando Eötvös Nominatae, Sectio computatorica, vol. 36, pp. 41-52, 2012.
[7] Á. Backhausz and T. F. Móri, "Weights and degrees in a random graph model based on 3-interactions," http://arxiv.org/abs/1206 .0633 .

[8] J. Neveu, Discrete-Parameter Martingales, North-Holland, Amsterdam, The Netherlands, 1975.

[9] A. P. Prudnikov, Yu. A. Brychkov, and O. I. Marichev, Integrals and Series, Gordon \& Breach Science Publishers, New York, NY, USA, 1986.

[10] V. V. Petrov, Sums of Independent Random Variables, Akademie, Berlin, Germany, 1975.

[11] W. Hoeffding, "Probability inequalities for sums of bounded random variables," Journal of the American Statistical Association, vol. 58, pp. 13-30, 1963.

[12] Á. Backhausz, Analysis of random graphs with methods of martingale theory [Ph.D. thesis], Eötvös Loránd University, Budapest, Hungary, 2012. 


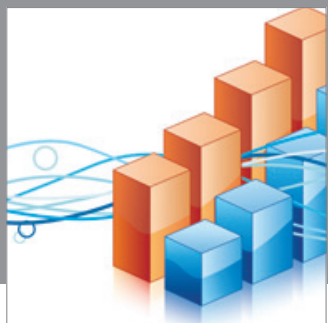

Advances in

Operations Research

mansans

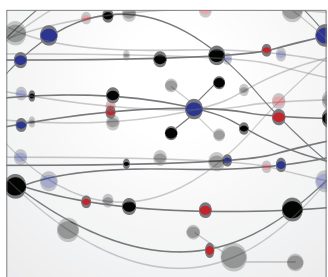

The Scientific World Journal
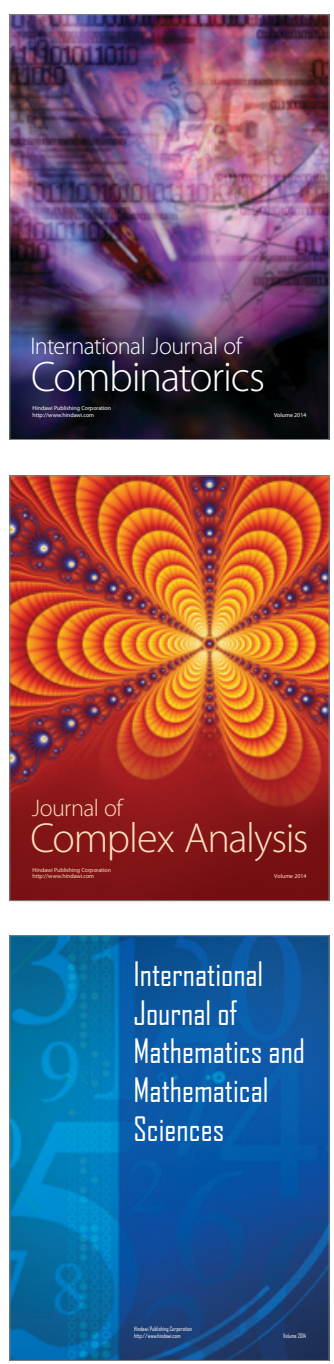
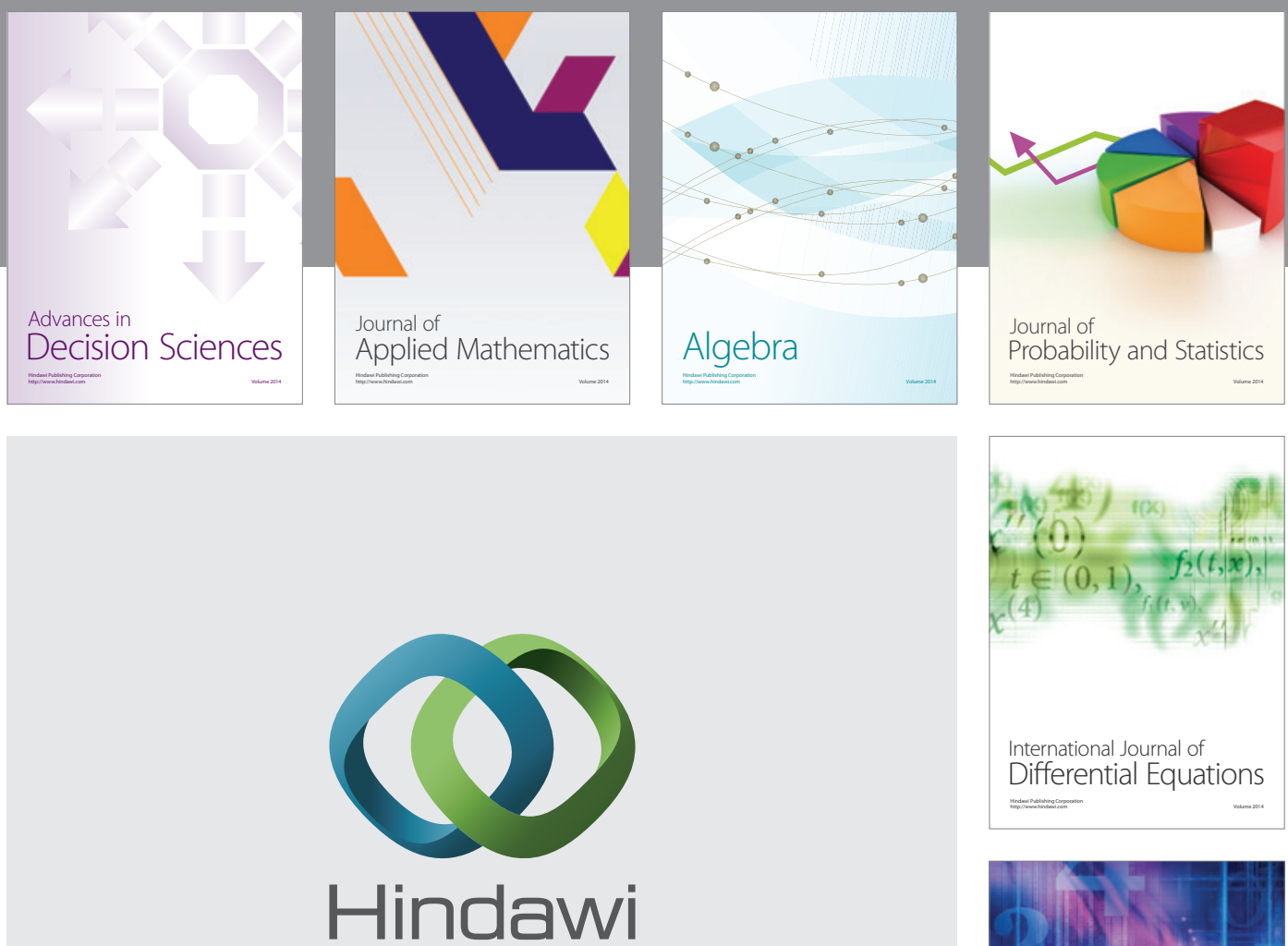

Submit your manuscripts at http://www.hindawi.com
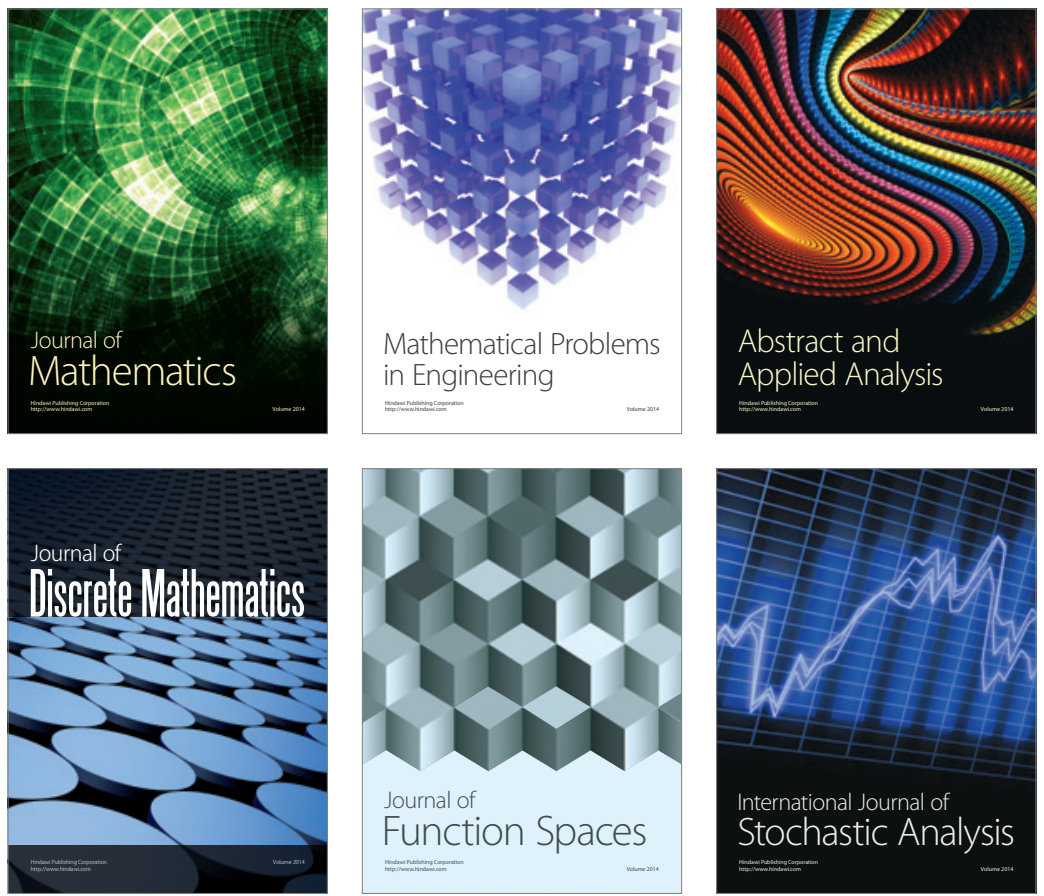

Journal of

Function Spaces

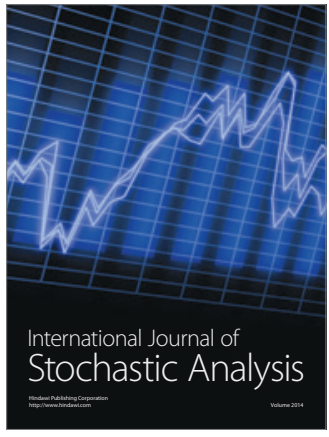

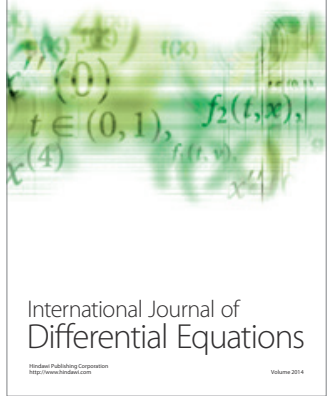
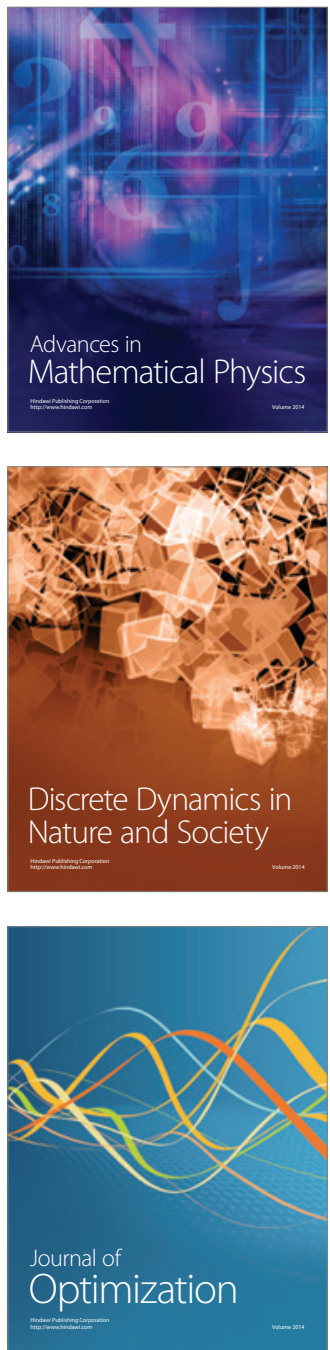